\title{
Percepções de Suporte Familiar e Qualidade de Vida entre Idosos Segundo a Vulnerabilidade Social
}

\author{
Relations between Social Vulnerability and Perceptions of Family Support \\ and Quality of Life among Elderly People
}

\author{
Keika Inouye*, Elizabeth Joan Barham, Elisete Silva Pedrazzani, \& Sofia Cristina Iost Pavarini \\ Universidade Federal de São Carlos, São Carlos, Brasil
}

\begin{abstract}
Resumo
No presente estudo, examinou-se a relação entre a percepção de qualidade de vida e de suporte familiar entre idosos, segundo seu nível de vulnerabilidade social e características sócio-demográficas. Foram entrevistados seis grupos de 25 idosos $(N=150)$, um grupo para cada nível de vulnerabilidade, em um município paulista de porte médio. Os instrumentos utilizados foram: Inventário de Percepção de Suporte Familiar, Escala de Avaliação da Qualidade de Vida - QdV-DA e o Critério de Classificação Econômica Brasil. Correlações estatisticamente significativas, indicaram que os níveis de vulnerabilidade social estavam diretamente relacionados a indicadores de nível socioeconômico, às percepções de suporte familiar (total, afetivo-consistente, de adaptação e autonomia) e de qualidade de vida.

Palavras-chave: Vulnerabilidade; Qualidade de Vida; Relações Familiares; Idosos; Envelhecimento.
\end{abstract}

\begin{abstract}
The present study examines the relation between perceptions of quality of life and of family support among elderly people according to their level of social vulnerability and other sociodemographic characteristics. Interviews were conducted with six groups of 25 elderly people $(N=150)$, one group for each level of vulnerability, in a medium-sized municipality in São Paulo state, Brazil. The instruments used were: the Family Support Perception Inventory, Quality of Life in Alzheimer's disease and the Brazilian Family Economic Classification Checklist. Statistically significant correlations indicate that levels of social vulnerability are directly related to socioeconomic measures, as expected, but also to perceptions of family support (total, consistent-affective, of adaptation and autonomy) and of quality of life.

Keywords: Vulnerability; Quality of Life; Family Relations; Elderly People; Aging.
\end{abstract}

As transformações decorrentes do envelhecimento têm despertado interesse crescente entre os pesquisadores no que se refere às questões ligadas ao bem-estar e à qualidade de vida na terceira idade (Freire, 2000). No contexto brasileiro atual, existem poucos serviços domiciliares especializados para apoiar as necessidades rotineiras de um idoso que acabam recebendo quase única e exclusivamente o suporte que seus familiares lhes oferecem. Esta situação pode ser desvantajosa, porque os mais velhos costumam ser pessoas de baixa prioridade na estrutura familiar, com os maiores investimentos de tempo e dinheiro focados nos mais jovens (Organização Mundial de Saúde [OMS], 2005).

Reconhecendo a importância da qualidade de vida para a manutenção da integridade física e mental das pessoas em todas as fases da vida, a OMS (2005) apóia atividades que permitam a obtenção de novos conhecimentos e

* Endereço para correspondência: Universidade Federal de São Carlos, Rodovia Washington Luis, Km 235, São Carlos, SP, Brasil, CEP 13565-905.E-mail: keikain@ terra.com.br a conseqüente otimização do bem-estar dos idosos. Atualmente, a qualidade de vida é definida como a percepção do indivíduo de sua posição na vida, no contexto da cultura e sistemas de valores nos quais vive e em relação aos seus objetivos, expectativas, padrões e preocupações. Esta definição inclui seis domínios principais: (a) saúde física, (b) estado psicológico, (c) níveis de independência, (d) relacionamento social, (e) meio-ambiente e (f) padrão espiritual (World Health Organization Quality of Life Working Group, 1995). Martin e Stockler (1998) sugerem que a qualidade de vida seja definida em termos da distância entre expectativas individuais e a realidade, sendo que quanto menor a distância, melhor. Embora o conceito de qualidade de vida tenha algumas variações, muitos pesquisadores (Fleck et al., 1999; Minayo, Hartz, \& Buss, 2000; Neri, 2006) concordam acerca de vários princípios que fazem parte de sua definição, tais como: (a) multidimensionalidade, relacionando-se ao fato de que a vida compreende múltiplas dimensões, tais como social, mental, material, física, cultural, econômica, dentre outras; (b) dinâmica, dada a sua 
característica inconstante no tempo e em diferentes espaços, como o trabalho, a família e a sociedade; (c) subjetividade, determinada pelo significado individual atribuído às experiências inter e intra-pessoais.

A dificuldade de se conceituar qualidade de vida está vinculada às percepções e atitudes singulares que cada indivíduo tem diante das conquistas, perdas e adversidades. Os parâmetros quantitativos e objetivos de saúde, de recursos econômicos e de interações sociais são menos relevantes, e o que assume maior importância é equilíbrio entre as limitações e as potencialidades que permitem compensações e adaptações diante das mudanças do meio (Chachamovich, Trentini, \& Fleck, 2007; Neri, 2007). Do ponto de vista teórico, a qualidade de vida é permeada por mecanismos de auto-regulação e pelo senso de ajustamento psicológico em interação com os recursos sociais e os valores culturais. Sendo assim, está no âmbito da experiência privada, que inclui competências comportamentais e avaliações comparativas entre expectativas e situações concretas, ambas em constantes transformações (Neri, 2007).

Uma das estratégias consideradas importantes para um envelhecimento com qualidade de vida é uma postura pró-ativa, de forma que o idoso deva escolher seus próprios objetivos e lutar para alcançá-los, reunindo recursos que são úteis na adaptação às mudanças e ativamente envolvido na manutenção do seu bem-estar. Além disso, os modelos de qualidade de vida usam conceitos de independência, controle, competências sociais e cognitivas (Neri, 2006; Sousa, Galante, \& Figueredo, 2003). Segundo Fleck, Chachamovich e Trentini (2003), as pesquisas sobre as condições que levam à uma boa qualidade de vida na velhice estão permitindo melhorias em práticas cotidianas, com impactos sociais positivos.

Tendo em vista a relação com fatores culturais, faz-se necessário conhecer a forma como a população de interesse conceitua qualidade de vida. Segundo um levantamento realizado por Paschoal, Jacob Filho e Litvoc (2008) por meio de entrevistas com 193 idosos na cidade de São Paulo/SP, a qualidade de vida desta população era composta por oito dimensões principais. São elas: saúde física, capacidade funcional e autonomia, psicológica, social e familiar, econômica, hábitos e estilo de vida, meio ambiente, espiritualidade e transcendência. Em estudo semelhante com 38 sujeitos com idade de 55 anos ou mais, Pereira (2002) enumerou itens que estes relataram como importantes para a qualidade de vida. Os participantes intercalaram necessidades espirituais e cognitivas com necessidades de subsistência e convívio social. Dentre os itens priorizados estavam a religiosidade, a família, a situação financeira, a saúde, os amigos e a ocupação. Estes estudos apontam algumas variáveis importantes para a qualidade de vida na velhice no Brasil, que coincidem com as descritas pela OMS (2005), dentre elas destacou-se para esta pesquisa, a situação de vulnerabilidade social, que abrange questões sócio-econômicas individuais e coletivas (Fundação SEADE, n.d.), e a percepção do suporte familiar, que faz parte das dimensões psicológicas, familiares e de convívio.

Em nosso contexto, a família geralmente constitui a principal rede de apoio social, exercendo função protetora diante das tensões geradas pela vida cotidiana (Bernal, 2003). O suporte familiar pode ser definido como o recurso primário de socialização por meio do qual os padrões de comportamento, hábitos de linguagem, valores, crenças e costumes são transmitidos e as necessidades psicológicas e fisiológicas básicas podem ser supridas (Gomes, 1994; Neri, 2005). Um suporte familiar adequado resulta em efeitos emocionais positivos e em sensações de pertencimento, cuidado e estima. Trata-se de um processo recíproco entre os membros da família, que gera efeitos tanto para quem recebe, como também para quem oferece o apoio (Williams \& Aiello, 2004).

Sabe-se que o vínculo e o apego entre familiares são vitais para o desenvolvimento do ser humano e são essas vivências que servirão de alicerce para a formação das percepções e capacidades interpessoais de todos os membros, mesmo durante a velhice. Mais especificamente, a percepção de suporte familiar está relacionada à competência social, capacidade de enfrentamento de problemas, percepção de controle, senso de estabilidade, autoconceito, afeto e, por conseqüência, ao bem-estar psicológico. $\mathrm{O}$ bem-estar psicológico pode ser definido como as crenças pessoais sobre o cumprimento de normas e papéis relacionados ao gênero e a idade, ao envolvimento com os outros, à produtividade, ao reconhecimento social, à autonomia, ao domínio sobre o ambiente, ao valor das realizações, ao cumprimento de metas de vida e a outros aspectos que permitem ao indivíduo sentir-se bem consigo mesmo, quando se compara com outros de seu meio social (Neri, 2007).

Embora a estrutura familiar mais freqüentemente para o idoso seja a família multigeracional, não há garantia de que estas estejam preparadas para assumir o papel de cuidadora (Cattani \& Girardon-Perlini, 2004). As famílias estão menores e com um maior número de idosos em sua composição, isso significa uma nova demanda de convivência (Camarano, Pasinato, \& Lemos, 2007). Dentro do contexto familiar, existem relações afetivas e pessoais na construção histórica de cada um de seus membros, que precisam ser analisadas, dentre elas, um fato relevante é a atribuição cultural da mulher como a principal provedora de cuidados no contexto da família (Karsch, 2003), que leva a hipótese de que idosos que moram com filhas dispõem de melhor suporte familiar.

O construto suporte familiar baseia-se em três dimensões principais: coesão, adaptabilidade e comunicação. A dimensão de coesão familiar diz respeito ao grau de separação e conexão entre os membros da família, abordando o vínculo emocional que seus integrantes possuem uns com os outros, sendo composta pelas coalizões internas entre os membros, os vínculos ou ligações, amizade, 
interesses em comum e recreação. A adaptabilidade se refere à capacidade da família de ser flexível para lidar com mudanças, por meio de alterações na estrutura de poder e nas regras de relacionamento em função do desenvolvimento de capacidades novas ou como resposta a obstáculos ou eventos estressantes que atingem seus membros. As reações da família dependem de habilidades como assertividade, controle e disciplina, além da capacidade para negociar estilos e regras. Por último, a comunicação familiar também é facilitada pelas habilidades interpessoais dos membros na expressão de afeto, com destaque para a empatia, escuta reflexiva, comentários suportivos e a capacidade de dividir seus sentimentos uns com os outros. Em contraposição, a comunicação negativa resultaria em altos graus de criticismo, além de mensagens dúbias entre os familiares (Baptista, 2005).

Predominantemente, os autores defendem que o suporte familiar compartilhado entre os indivíduos traz benefícios para todos, tais como a minimização do estresse, melhoria da auto-estima e da saúde, influenciando positivamente no bem-estar psicológico (Souza, Mello, \& Rezende, 2008). Contudo, existem autores que apontam resultados negativos do suporte familiar em função de situações que sobrecarregam o cuidador familiar ou quando há uma excessiva assistência, resultando em falta de liberdade e autonomia tanto para o cuidador como para o receptor de ajuda desnecessária. Assim, nem sempre a frequiência das relações sociais dentro da família denota qualidade de suporte familiar de forma balanceada e saudável (Ramos, 2007).

A relação cuidador familiar e idoso é importante para a manutenção da qualidade de vida de quem está recebendo cuidados, porém um idoso no convívio multigeracional é uma situação delicada, pois pode afetar a vida familiar, na área financeira, de exercício de papéis e das relações com amigos e vizinhos. As exigências, demandas ou pressões geram comportamentos emocionais que incluem ações verbais e motoras tanto de acolhimento quanto de exclusão que geram no idoso sentimentos de conforto ou estresse (Inouye, 2008). Devido ao declínio das funções orgânicas que conduzem à perda do vigor físico, comprometimento do sistema imunológico e diminuição da capacidade adaptativa às variações do meio interno e externo, alguns autores consideram o processo de envelhecimento em si uma condição de vulnerabilidade (Fried et al., 2001).

A vulnerabilidade social, em termos conceituais, é uma síndrome multidimensional, resultante de carências econômicas, sociais, psicológicas, cognitivas ou físicas, que culmina em um estado de maior risco para a ocorrência de desfechos clínicos adversos, deterioração do nível de bem-estar, hospitalização, institucionalização e morte (Teixeira, 2006). Segundo Busso (2001), entre os fatores que compõem as situações de vulnerabilidade social estão: (a) a fragilidade ou desproteção frente às mudanças; (b) a debilidade interna de indivíduos para realizar as mudanças necessárias a fim de aproveitar os recursos que estão ao seu alcance; (c) a insegurança que desmotiva ações com o objetivo de lograr melhores condições de vida. Desta forma, a vulnerabilidade de um indivíduo ou grupo refere-se à sua capacidade de controlar as forças que afetam seu bem-estar, a fim de aproveitar ativamente as oportunidades propiciadas pelo ambiente (Kaztman, 2000).

O Índice Paulista de Vulnerabilidade Social (IPVS), utilizado para categorizar os sujeitos desta pesquisa, é um indicador de vulnerabilidade produzido no âmbito do Censo Demográfico de 2000 pela Fundação SEADE. Este indicador consiste em uma tipologia derivada da combinação entre dois fatores:

Fator Socioeconômico. As variáveis mais importantes na sua composição são: (a) porcentagem de responsáveis pelo domicílio alfabetizados no total de responsáveis do setor censitário; (b) porcentagem de responsáveis pelo domicílio com ensino fundamental completo no total de responsáveis do setor censitário; (c) anos médios de estudo do responsável pelo domicílio; (d) rendimento nominal médio do responsável pelo domicílio; (e) porcentagem de responsáveis com rendimento de até 3 salários mínimos no total de responsáveis do setor censitário.

Fator Ciclo de Vida Familiar. As variáveis mais importantes na sua composição são: (a) porcentagem de responsáveis pelo domicílio com idade entre 10 e 29 anos no total de responsáveis do setor censitário; (b) idade média do responsável pelo domicílio; (c) porcentagem de crianças de 0 a 4 anos no total da população do setor censitário.

A combinação destes dois fatores resulta em seis grupos de vulnerabilidade social assim denominados: (a) Grupo 1: Nenhuma vulnerabilidade; (b) Grupo 2: Vulnerabilidade muito baixa; (c) Grupo 3: Vulnerabilidade baixa; (d) Grupo 4: Vulnerabilidade média; (e) Grupo 5: Vulnerabilidade alta; (f) Grupo 6: Vulnerabilidade muito alta. Os critérios que definiram a escala de vulnerabilidade são relativos à realidade do Estado de São Paulo em 2000. A condição de "nenhuma vulnerabilidade" corresponde à melhor situação observada no Estado naquele ano e a de "vulnerabilidade muito alta" à pior (Fundação SEADE, n.d.).

Embora os estudos descritivos apontem possíveis relações entre as variáveis qualidade de vida, percepção de suporte familiar e vulnerabilidade social, não foram encontradas pesquisas que correlacionassem tais variáveis a fim de se confirmar estas associações e estabelecer em que intensidade estas ocorrem. Diante do panorama traçado a partir dos estudos realizados até o momento, os objetivos deste trabalho foram: (a) descrever o perfil sócio-demográfico, a percepção de qualidade de vida e de suporte familiar de uma amostra de idosos em seis níveis de vulnerabilidade social, segundo o IPVS; (b) identificar diferenças na percepção de qualidade de vida e suporte familiar entre os seis diferentes níveis de vulnerabilidade 
social; (c) relacionar variáveis sócio-demográficas com os escores percepção de qualidade de vida e de suporte familiar; (d) relacionar as variáveis nível de vulnerabilidade social, percepção de qualidade de vida e suporte familiar de idosos entre si.

\section{Método}

\section{Local, Período do Estudo e Participantes}

O presente estudo foi realizado em São Carlos (SP), um município de porte médio, situado na região central do estado de São Paulo e dividido pelo Instituto Brasileiro de Geografia e Estatística (IBGE) em 267 setores censitários (territórios contíguos com relativa homogeneidade socioeconômica que abrigam em média 300 domicílios). Segundo dados da mesma instituição, em 2007, a população deste município era de aproximadamente 213 mil habitantes, $12 \%$ dos quais apresentavam 60 anos ou mais (IBGE, 2007). A coleta de dados teve duração de 5 meses, com início em junho de 2008, estendendo-se até outubro de 2008.

Para cada um dos seis níveis de vulnerabilidade (nenhuma vulnerabilidade; vulnerabilidade muito baixa; vulnerabilidade baixa; vulnerabilidade média; vulnerabilidade alta; vulnerabilidade muito alta), foram entrevistados 25 idosos de um setor censitário determinado por meio de sorteio. A amostra total foi composta por 150 sujeitos de pesquisa, que atenderam aos seguintes critérios de inclusão: (a) ter 60 anos ou mais, ou seja, ser considerado cronologicamente um idoso segundo critérios preconizados pela Organização Mundial de Saúde, (b) não apresentar distúrbios graves de linguagem, (c) aceitar pessoalmente participar do estudo de forma livre e esclarecida.

\section{Materiais e Instrumentos}

Para o desenvolvimento deste estudo foram utilizados os softwares estatísticos Statistical Package for Social Sciences - SPSS, versão 13.0 e STATÍSTICA, versão 7.0. No que se refere à divisão dos grupos segundo a variável vulnerabilidade social, foi adotado o Índice Paulista de Vulnerabilidade Social produzido no âmbito do Censo Demográfico 2000 (Fundação SEADE, n.d.) e, para caracterização dos sujeitos de pesquisa, foi utilizada uma "Ficha de Entrevista" para a obtenção de dados pessoais referentes à amostra (nome, localização residencial, gênero, idade, alfabetização/escolaridade, estado civil, número de filhos e integrantes do convívio familiar).

$\mathrm{O}$ instrumento de avaliação de qualidade de vida usado nesta pesquisa foi a "Escala de Qualidade de Vida" (QdV-DA), traduzida, adaptada transculturalmente (Novelli, Rovere, Nitrini, \& Caramelli, 2005) e validada para o uso no Brasil (Novelli, 2006). A escolha do instrumento deve-se ao fato deste ter sido originalmente construído por meio da revisão da literatura geriátrica e validado tanto para idosos com alterações cognitivas leves ou moderadas quanto para seus cuidadores, que não necessariamente apresentam problemas de saúde ou idade superior a 60 anos. Também, visto que na população mundial, as taxas médias de prevalência de demência variam de 1,17\% na faixa de 65-69 anos, a $54,83 \%$ na faixa acima de 95 anos (Lopes \& Bottino, 2002), o uso deste instrumento permitiu a entrevista aleatória de qualquer idoso da comunidade. Os treze itens da escala avaliados pelo participante são: saúde física, disposição, humor, moradia, memória, família, casamento, amigos, você em geral, capacidade de fazer tarefas, capacidade de fazer atividades de lazer, dinheiro e a vida em geral. Atribui-se pontuação 1 para "ruim", 2 para "regular", 3 para "bom" e 4 para "excelente", sendo a pontuação geral mínima de 13 e a máxima de 52 (Novelli, 2006).

O "Inventário de Percepção de Suporte Familiar" (IPSF), utilizado para mensurar a percepção de suporte familiar dos participantes, é composto por 42 questões, sendo todas fechadas, respondidas em uma escala tipo Likert de três pontos ("Quase Nunca ou Nunca”, “Às vezes" e "Quase Sempre ou Sempre"). O IPSF contempla três fatores: Afetivo-Consistente (21 itens), Adaptação (13 itens) e Autonomia Familiar (8 itens), uma vez que as pontuações de cada item variam de 0 a 2 , o escore total varia entre 0 e 84 pontos, sendo que pontuações mais altas indicam melhor percepção do suporte familiar (Baptista, 2005).

Para avaliar o nível socioeconômico de cada participante, o "Critério de Classificação Econômica Brasil" foi aplicado. Este instrumento estabelece faixas de poder aquisitivo familiar, baseando-se na quantidade de posse de bens de consumo duráveis, do grau de instrução do chefe da família e em alguns outros fatores como a presença de empregada doméstica. Esta escala divide a população em sete classes sociais (A1, A2, B1, B2, C, D e E). Os critérios para classificação social no Brasil foram estabelecidos pela Associação Brasileira de Anunciantes, pela Associação Nacional das Empresas de Pesquisa de Mercado e pela Associação Brasileira dos Institutos de Pesquisa de Mercado (Associação Brasileira de Empresas de Pesquisa [ABEP], 2003).

\section{Procedimentos de Coleta dos Dados}

Após aprovação do Comitê de Ética em Pesquisa (CEP) da Universidade Federal de São Carlos (UFSCar), foram sorteados os locais de residência (setores censitários) para coleta de dados. Os primeiros 25 concordantes de cada setor censitário que preenchiam os critérios de inclusão assinaram o Termo de Consentimento Livre e Esclarecido e responderam aos instrumentos citados no item anterior. Para cada sujeito de pesquisa, a coleta de dados foi realizada individualmente em um único encontro.

\section{Análise dos Dados}

Os escores foram padronizados para facilitar a compreensão das comparações entre os dados obtidos por diferentes instrumentos de medida. A padronização con- 
sistiu em dividir cada escore dos sujeitos pelo valor máximo da escala correspondente.

Os testes estatísticos utilizados para as comparações foram não-paramétricos, em virtude dos escores serem dados discretos ou não apresentarem distribuição normal. Foram realizadas: (a) análises estatísticas descritivas; (b) Teste de Kruskal-Wallis para variáveis divididas em três ou mais categorias independentes, seguido de teste post-hoc/comparações múltiplas; (c) Teste de MannWhitney para variáveis com duas categorias independentes; (d) correlação de Sperman. Foi considerado como ponto de corte para significância estatística o nível de $5 \%(p<0,05)$.

\section{Aspectos Éticos}

A coleta de dados foi iniciada somente após aprovação do Comitê de Ética em Pesquisa da UFSCar, com parecer número 233/2008. Todos os participantes foram informados dos objetivos do trabalho, consultados sobre a disponibilidade em participar do estudo e assegurados do sigilo das informações individuais. Somente após assinatura do "Termo de Consentimento Livre e Esclarecido" os participantes foram entrevistados. Nenhum sujeito foi exposto a atividades que ocasionassem desconforto, humilhação ou risco. Não se observou nenhum tipo de dano com a aplicação dos instrumentos. Foi assegurado ao participante, a qualquer momento durante a coleta de dados, o direito de interromper sua participação sem compromisso de justificativa.

\section{Resultados}

O gênero da amostra estudada foi predominantemente feminino $(64 \%, n=96)$, oriundas das classes B1 e B2 $(8 \%, n=12), \mathrm{C}(33 \%, n=49), \mathrm{D}(55 \%, n=82)$ ou $\mathrm{E}$ $(5 \%, n=7)$; não foi entrevistado nenhum sujeito das classes A1 e A2. A média das idades de ambos os gêneros foi de 72,3 anos $(D P=7,9$, Mín. = 60, Máx. $=93)$, com mulheres tendo uma média de 72,9 anos $(D P=7,8$, Mín. = 60, Máx. = 93 $)$ e os homens, de 71,4 anos $(D P=$ 8,2 , Mín. =60, Máx. =92). No que se refere à alfabetização, $61 \%(n=92)$ afirmaram saber ler e escrever $\mathrm{e}$ $39 \%(n=58)$ eram analfabetos.

Os dados referentes ao estado conjugal mostraram que $41 \%(n=61)$ dos sujeitos viviam com um companheiro e $59 \%(n=89)$ eram viúvos, separados ou solteiros. O número mediano de filhos $\left(Q_{2}\right.$ ou segundo quartil) foi de 4 (Mín. = 0, Máx. $=12)$, sendo $Q_{1}$ (primeiro quartil) $=2$ e $Q_{3}$ (terceiro quartil) $=7$. Quanto ao número de integrantes do convívio familiar que dividiam a mesma residência, 23\% dos idosos $(n=34)$ moravam sozinhos enquanto $77 \%(n=116)$ dividiam a casa com outras pessoas como esposo(a) ou companheiro(a) $(77 \%, n=116)$, filhos ( $35 \%, n=52)$, filhas ( $22 \%, n=33)$, netos(as) (29\%, $n=43)$ e/ou outros $(24 \%, n=36)$.

A mediana $\left(Q_{2}\right)$ dos escores totais padronizados de qualidade de vida foi de 77 pontos (Mín. = 35, Máx. =
100 ), sendo $Q_{1}=65$ e $Q_{3}=90$. No que se refere aos escores totais padronizados de percepção de suporte familiar, a mediana $\left(Q_{2}\right)$ encontrada foi 83 pontos (Mín. = 29 , Máx. $=100$ ), sendo $Q_{1}=70$ e $Q_{3}=92$. Os fatores afetivo consistente, adaptação e autonomia familiar apresentaram as seguintes medianas $\left(Q_{2}\right) 74$ (Mín. $=12$, Máx. = 100), 96 (Mín. = 4, Máx. = 100) e 100 pontos (Mín. = 13, Máx. $=100)$, respectivamente.

Os resultados descritivos obtidos para as variáveis sócio-demográficas, bem como a análise de significância de sua influência nos escores de qualidade de vida e nos escores de percepção de suporte familiar são apresentados nas Tabelas 1 e 2, respectivamente, utilizando o Teste de Mann-Whitney $(Z)$ ou Kruskal-Wallis $\left(\chi^{2}\right)^{*}$ e Teste Correlacional de Spearman.

A partir dos dados das Tabelas 1 e 2 verificou-se que: o nível sócio-econômico interfere nos escores de qualidade de vida e de percepção de suporte familiar total, bem como nos fatores de percepção afetivo consistente, de adaptação e de autonomia familiar $(p<0,05)$, sendo que as classes $\mathrm{B} / \mathrm{C}$ apresentaram escores superiores às classes D/E. Isto também é confirmado pela análise de correlação entre os escores e a pontuação na classificação econômica, que apesar de moderadas ou fracas foram significativas e positivas.

$\mathrm{O}$ fato dos idosos saberem ler/escrever também influencia os escores mencionados significativamente $(p<$ $0,05)$, sendo que aqueles que tinham habilidades de leitura e escrita apresentaram escores medianos superiores aos que disseram não saber ler/escrever. E também neste caso, as correlações do grau de escolaridade com os escores foram moderadas ou fracas, mas significativas e positivas.

Os idosos que afirmaram morar com netos e/ou outros parentes apresentaram escores medianos de qualidade de vida inferiores aos que disseram não morar com netos e/ ou outros parentes $(p<0,05)$; para os outros escores de percepção de suporte familiar não ocorreram diferenças significativas e, neste caso, é importante mencionar que os outros parentes citados são em $38 \%$ dos casos os genros e em $34 \%$ as noras;

As correlações dos escores com o número de pessoas na casa não foram significativas, mas as correlações dos escores com o número de filhas ou filhos foram significativas e negativas, porém fracas, exceto para a correlação entre o escore de autonomia familiar com o número de filhos que não foi significativo.

Para as demais variáveis testadas (sexo, faixa-etária, possuir companheiro, morar sozinho, possuir filhos, morar com filhos ou filhas) não foram encontradas diferenças significativas entre os grupos nos escores de qualidade de vida e de percepção do suporte familiar. Também não ocorreram correlações significativas entre tempo de vida conjugal e os escores de qualidade de vida e de percepção de suporte familiar.

Ao avaliar as correlações entre os escores de qualidade de vida e de percepção de suporte familiar total, afetivo 
Inouye, K., Barham, E. J., Pedrazzani, E. S. \& Pavarini, S. C. I. (2010). Percepções de Suporte Familiar e Qualidade de Vida entre Idosos Segundo a Vulnerabilidade Social.

Tabela 1

Valores Medianos $\left(Q_{2}\right)$ dos Escores Totais Padronizados de Qualidade de Vida $(Q V)$, Percepção de Suporte Familiar Total (IPSF-T), Afetivo Consistente (IPSF-AFET), de Adaptação (IPSF-ADAP) e Autonomia (APSF-AUT) segundo as Variáveis Sócio-demográficas Categóricas Examinadas Junto aos Idosos Entrevistados

\begin{tabular}{|c|c|c|c|c|c|c|c|}
\hline \multirow[t]{2}{*}{ Variáveis sócio-demográficas } & \multirow[t]{2}{*}{ Freq. } & \multicolumn{6}{|c|}{ Mediana $\left(Q_{2}\right)$ dos escores padronizados } \\
\hline & & $n$ & QV & IPSF-T & IPSF-AFET & IPSF-ADAP & APSF-AUT \\
\hline \multirow[t]{2}{*}{ Sexo } & Masc. & 54 & 73 & 81 & 71 & 96 & 97 \\
\hline & Fem. & 96 & 79 & 84 & 74 & 100 & 100 \\
\hline \multirow[t]{5}{*}{ Faixa etária $(\text { anos })^{*}$} & $60-65$ & 35 & 73 & 81 & 71 & 92 & 100 \\
\hline & $66-70$ & 30 & 88 & 85 & 82 & 100 & 100 \\
\hline & $71-75$ & 36 & 79 & 81 & 71 & 96 & 100 \\
\hline & $76-80$ & 28 & 72 & 82 & 74 & 98 & 88 \\
\hline & + de 81 & 21 & 81 & 85 & 79 & 100 & 94 \\
\hline \multirow[t]{2}{*}{ Nível socioeconômico } & $\mathrm{B} / \mathrm{C}$ & 61 & $90^{c}$ & $92^{c}$ & $86^{c}$ & $100^{\mathrm{c}}$ & $100^{\mathrm{c}}$ \\
\hline & $\mathrm{D} / \mathrm{E}$ & 89 & 73 & 75 & 67 & 88 & 94 \\
\hline \multirow[t]{2}{*}{ Ler e escrever } & Sabe & 92 & $87^{c}$ & $88^{c}$ & $81^{c}$ & $100^{\mathrm{c}}$ & $100^{\mathrm{c}}$ \\
\hline & Não sabe & 58 & 65 & 72 & 63 & 88 & 88 \\
\hline \multirow[t]{2}{*}{ Companheiro (conjugal) } & Tem & 61 & 79 & 82 & 76 & 96 & 100 \\
\hline & Não tem & 89 & 75 & 83 & 74 & 96 & 100 \\
\hline \multirow[t]{2}{*}{ Como vive } & Sozinho & 34 & 85 & 86 & 71 & 100 & 100 \\
\hline & Com outros & 116 & 74 & 81 & 74 & 96 & 100 \\
\hline \multirow[t]{2}{*}{ Filhos } & Têm & 138 & 75 & 82 & 73 & 96 & 100 \\
\hline & Não tem & 12 & 89 & 88 & 82 & 98 & 100 \\
\hline \multirow[t]{2}{*}{ Mora com filhos } & Sim & 52 & 71 & 79 & 69 & 94 & 100 \\
\hline & Não & 98 & 81 & 85 & 77 & 100 & 100 \\
\hline \multirow[t]{2}{*}{ Mora com filhas } & Sim & 33 & 75 & 83 & 74 & 96 & 94 \\
\hline & Não & 117 & 79 & 82 & 71 & 96 & 100 \\
\hline \multirow[t]{2}{*}{ Mora com netos } & Sim & 43 & $73^{a}$ & 77 & 74 & 92 & 100 \\
\hline & Não & 107 & 83 & 85 & 71 & 100 & 100 \\
\hline \multirow[t]{2}{*}{ Mora com outros } & Sim & 32 & $69^{a}$ & 75 & 73 & 96 & 100 \\
\hline & Não & 118 & 80 & 84 & 74 & 96 & 100 \\
\hline
\end{tabular}

Nota.${ }^{\text {a }}$ diferença significativa, $p<0,05 ;{ }^{\text {b }}$ diferença significativa, $p<0,01 ;{ }^{\text {c }}$ diferença significativa, $p<0,001 ; *$ Teste de Kruskal-Wallis $\left(\chi^{2}\right)$.

Tabela 2

Resultados das Análises dos Escores Totais Padronizados de Qualidade de Vida (QV), Percepção de Suporte Familiar Total (IPSF-T), Afetivo Consistente (IPSF-AFET), de Adaptação (IPSF-ADAP) e Autonomia (APSFAUT) segundo as Variáveis Sócio-demográficas dos Idosos Entrevistados por Meio do Teste Correlacional de Spearman (rho)

\begin{tabular}{lccccc}
\hline Variáveis & QV & IPSF-AFET & IPSF-ADAP & IPSF-AUT & \multicolumn{2}{c}{ IPSF-T } \\
\hline Pontuação Critério Brasil & $0,443^{\mathrm{c}}$ & $0,555^{\mathrm{c}}$ & $0,472^{\mathrm{c}}$ & $0,279^{\mathrm{b}}$ & $0,569^{\mathrm{c}}$ \\
Idade & $0,009^{\mathrm{c}}$ & $0,112^{\mathrm{a}}$ & 0,068 & $-0,262^{\mathrm{b}}$ & 0,028 \\
Grau de escolaridade & $0,421^{\mathrm{c}}$ & $0,363^{\mathrm{c}}$ & $0,333^{\mathrm{c}}$ & $0,212^{\mathrm{b}}$ & $0,387^{\mathrm{c}}$ \\
Tempo de vida conjugal & $0,072^{\mathrm{a}}$ & 0,019 & $-0,061$ & $-0,030$ & 0,029 \\
Número total de filhos & $-0,123$ & $-0,036$ & $-0,041$ & $-0,078$ & $-0,068$ \\
Número de filhos & $-0,246^{\mathrm{b}}$ & $-0,266^{\mathrm{b}}$ & $-0,232^{\mathrm{b}}$ & $-0,067$ & $-0,263^{\mathrm{b}}$ \\
Número de filhas & $-0,338^{\mathrm{c}}$ & $-0,244^{\mathrm{b}}$ & $-0,265^{\mathrm{b}}$ & $-0,283^{\mathrm{c}}$ & $-0,317^{\mathrm{c}}$ \\
Número de pessoas na casa & $-0,071$ & 0,048 & $-0,077$ & $-0,126$ & $-0,013$ \\
\hline
\end{tabular}

Nota. ${ }^{\text {a }}$ diferença significativa, $p<0,05 ;{ }^{\mathrm{b}}$ diferença significativa, $p<0,01 ;{ }^{\mathrm{c}}$ diferença significativa, $p<0,001$. 
consistente, de adaptação e de autonomia, verificou-se que estas foram significativas $(p<0,05)$ e positivas, porém em intensidades diferentes: (a) fortes entre o escore de qualidade de vida com o escore de percepção de suporte familiar afetivo consistente $(r=0,721)$ e o escore total de percepção de suporte familiar $(r=0,794)$; (b) moderadas entre o escore de qualidade de vida com os escores de percepção de adaptação familiar $(r=0,636) \mathrm{e}$ autonomia familiar $(r=0,482)$.
Nas comparações dos subgrupos da amostra dividida em seis diferentes níveis de vulnerabilidade social, segundo o Índice Paulista de Vulnerabilidade Social (IPVS), as descrições comparativas das variáveis sócio-demográficas, bem como os escores medianos $\left(\mathrm{Q}_{2}\right)$, os escores mínimos e máximos de qualidade de vida e de percepção de suporte familiar total (IPSF-T), afetivo consistente (IPSF-AFET), de adaptação (IPSF-ADAP) e autonomia (APSF-AUT) são apresentados na Tabela 3.

Tabela 3

Distribuição das Variáveis Sócio-demográficas (em porcentagem), dos Escores Medianos $\left(Q_{2}\right)$, dos Escores Mínimos e Máximos de Qualidade de Vida (QV) e de Percepção de Suporte Familiar Total (IPSF-T), Afetivo Consistente (IPSF-AFET), de Adaptação (IPSF-ADAP) e Autonomia (APSF-AUT) dos Subgrupos da Amostra Dividida em Seis Diferentes Níveis de Vulnerabilidade Social, segundo o Índice Paulista de Vulnerabilidade Social (IPVS)

\begin{tabular}{lrrrrrr}
\hline Variáveis & IPVS 1 & IPVS 2 & IPVS 3 & IPVS 4 & IPVS 5 & IPVS 6 \\
\hline Sexo (Percentual de mulheres) & $64 \%$ & $80 \%$ & $64 \%$ & $60 \%$ & $64 \%$ & $52 \%$ \\
Idade mediana (anos) & 72 & 75 & 71 & 72 & 70 & 70 \\
Sabe ler/escrever & $88 \%$ & $88 \%$ & $48 \%$ & $52 \%$ & $52 \%$ & $40 \%$ \\
Casados/tem companheiro & $48 \%$ & $20 \%$ & $52 \%$ & $48 \%$ & $40 \%$ & $36 \%$ \\
Mora sozinho(a) & $32 \%$ & $32 \%$ & $28 \%$ & $8 \%$ & $16 \%$ & $20 \%$ \\
Número mediano de filhos & 3 & 2 & 5 & 7 & 6 & 5 \\
Classe Social menos favorecida (D/E)* & $28 \%$ & $52 \%$ & $72 \%$ & $44 \%$ & $80 \%$ & $80 \%$ \\
Escore mediano de QV $\left(Q_{2}\right)$ & 94 & 92 & 87 & 67 & 67 & 65 \\
Escore mediano do IPSF-T $\left(Q_{2}\right)$ & 96 & 90 & 86 & 79 & 70 & 55 \\
Escore mediano do IPSF-AFET $\left(Q_{2}\right)$ & 93 & 83 & 76 & 67 & 57 & 50 \\
Escore mediano do IPSF-ADAP $\left(Q_{2}\right)$ & 100 & 100 & 96 & 96 & 88 & 69 \\
Escore mediano do APSF-AUT $\left(Q_{2}\right)$ & 100 & 100 & 100 & 100 & 63 & 56 \\
\hline
\end{tabular}

Nota. *Vale lembrar que em cada grupo de vulnerabilidade social residem indivíduos de com diferentes níveis de poder aquisitivo segundo o Critério de Classificação Econômica Brasil.

Tabela 4

Teste de Comparações Múltiplas para Identificação das Diferenças entre os Níveis de Vulnerabilidade Social (IPVS)

\begin{tabular}{|c|c|c|c|c|c|}
\hline \multirow[t]{2}{*}{ Comparações Múltiplas entre } & QV & IPSF-T & IPSF-AFET & IPSF-ADAP & APSF-AUT \\
\hline & $Z$ & $Z$ & $Z$ & $Z$ & $Z$ \\
\hline IPVS 1 e IPVS 2 & 1,131 & 1,589 & 1,597 & 0,435 & 1,274 \\
\hline IPVS 1 e IPVS 3 & $2,933^{a}$ & $3,171^{\mathrm{a}}$ & 2,920 & $3,104^{\mathrm{a}}$ & 1,172 \\
\hline IPVS 1 e IPVS 4 & $5,825^{\mathrm{c}}$ & $4,639^{\mathrm{c}}$ & $4,634^{\mathrm{c}}$ & 2,902 & 2,176 \\
\hline IPVS 1 e IPVS 5 & $6,896^{c}$ & $6,922^{c}$ & $6,169^{c}$ & $4,373^{c}$ & $4,577^{\mathrm{c}}$ \\
\hline IPVS 1 e IPVS 6 & $6,955^{\mathrm{c}}$ & $7,635^{\mathrm{c}}$ & $6,741^{\mathrm{c}}$ & $5,983^{\mathrm{c}}$ & $4,971^{\mathrm{c}}$ \\
\hline IPVS 2 e IPVS 3 & 1,802 & 1,582 & 1,323 & 2,669 & 0,103 \\
\hline IPVS 2 e IPVS 4 & $4,694^{\mathrm{c}}$ & $3,050^{\mathrm{a}}$ & $3,037^{\text {a }}$ & 2,467 & 0,902 \\
\hline IPVS 2 e IPVS 5 & $5,765^{\mathrm{c}}$ & $5,334^{\mathrm{c}}$ & $4,572^{c}$ & $3,939^{\mathrm{c}}$ & $3,302^{a}$ \\
\hline IPVS 2 e IPVS 6 & $5,823^{\mathrm{c}}$ & $6,046^{c}$ & $5,145^{\mathrm{c}}$ & $5,548^{c}$ & $3,696^{\mathrm{b}}$ \\
\hline IPVS 3 e IPVS 4 & 2,892 & 1,468 & 1,714 & 0,202 & 1,004 \\
\hline IPVS 3 e IPVS 5 & $3,963^{\mathrm{c}}$ & $3,752^{\mathrm{b}}$ & $3,249^{\mathrm{a}}$ & 1,270 & $3,405^{\text {a }}$ \\
\hline IPVS 3 e IPVS 6 & $4,022^{\mathrm{c}}$ & $4,464^{\mathrm{c}}$ & $3,822^{b}$ & 2,879 & $3,799^{b}$ \\
\hline IPVS 4 e IPVS 5 & 1,071 & 2,283 & 1,535 & 1,471 & 2,401 \\
\hline IPVS 4 e IPVS 6 & 1,130 & $2,996^{\mathrm{a}}$ & 2,108 & $3,081^{\text {a }}$ & 2,795 \\
\hline IPVS 5 e IPVS 6 & 0,059 & 0,713 & 0,573 & 1,610 & 0,394 \\
\hline
\end{tabular}

Nota. ${ }^{\text {a }}$ diferença significativa, $p<0,05 ;{ }^{\text {b }}$ diferença significativa, $p<0,01 ;{ }^{\mathrm{c}}$ diferença significativa, $p<0,001$. 
Por meio do teste de Kruskal-Wallis, verificou-se que existe pelo menos uma diferença entre os diferentes níveis de vulnerabilidade social em relação aos escores de qualidade de vida $\left(\chi^{2}=91,884 ; d f=5 ; p<0,001\right)$, de percepção de suporte familiar total (IPSF-T) $\left(\chi^{2}=89,546\right.$; $d f=5 ; p<0,001)$, afetivo consistente (IPSF-AFET) $\left(\chi^{2}\right.$ $=70,146 ; d f=5 ; p<0,001$ ), de adaptação (IPSF-ADAP) $\left(\chi^{2}=58,656 ; d f=5 ; p<0,001\right)$ e autonomia (APSFAUT) $\left(\chi^{2}=48,068 ; d f=5 ; p<0,001\right)$. Então, para identificar as diferenças apontadas pelo teste de Kruskal-Wallis foi aplicado o teste de comparações múltiplas (Tabela 4).

Os resultados destas comparações múltiplas apontam que: para a qualidade de vida, os escores dos IPVS's 1 e 2 são significativamente superiores aos dos IPVS's 4,5 e 6, do mesmo modo, o escore do IPVS 3 é superior aos dos IPVS's 5 e 6. Os escores dos IPVS's 1, 2 e 3 não são diferentes entre si e os escores dos IPVS's 4, 5 e 6 e dos IPVS's 3 e 4 também não. As correlações, todas negativas entre IPVS e os itens da escala, evidenciam que à medida que aumenta a vulnerabilidade social piora a percepção da qualidade de vida pelo idoso, em todos os itens da escala. Os itens mais afetados pelo nível de vulnerabilidade, em ordem decrescente de coeficiente de correlação, foram: humor $(r=-0,765, p<0,001)$, moradia $(r=-0,765, p<0,001)$, casamento $(r=-0,741, p<$ $0,001)$, família $(r=-0,736, p<0,001)$ e você em geral $(r$ $=-0,579, p<0,001)$.

Para a percepção de suporte familiar, os escores dos IPVS's 1 e 2 são superiores aos dos IPVS's 4, 5 e 6, o mesmo ocorre com o IPVS 3 em relação aos IPVS's 5 e 6. Os escores dos IPVS's 1 e 2, IPVS's 3 e 4, IPVS's 4 e 5 e IPVS's 5 e 6 não são significativamente diferentes entre si. As correlações negativas apontam que à medida que aumenta a vulnerabilidade social piora a percepção geral de suporte familiar.

Para a percepção de suporte familiar afetivo consistente, os escores dos IPVS's 1 e 2 são significativamente superiores aos dos IPVS's 4, 5 e 6, o mesmo ocorre com o IPVS 3 em relação aos IPVS's 5 e 6. Os escores dos IPVS's 1, 2 e 3, IPVS's 4, 5 e 6 e IPVS's 3 e 4 não são significativamente diferentes entre si. Os itens da escala mais afetados pelo nível de vulnerabilidade, em ordem decrescente de coeficiente de correlação, foram os relacionados à: receber elogios $(r=-0,578, p<0,001)$; expressão de pensamentos e emoções $(r=-0,528, p<0,001)$, discussão dos medos e preocupações $(r=-0,567, p<$ $0,001)$.

Os escores de adaptação familiar do IPVS 1 são significativamente superiores aos dos IPVS's 3, 5 e 6; o escore do IPVS 2 é superior aos dos IPVS's 5 e 6; e o escore do IPVS 4 é superior ao do IPVS 6. Os itens da escala mais afetados pelo nível de vulnerabilidade foram, em ordem decrescente, aqueles relacionados à: irritação que a família causa no entrevistado $(r=-0,569, p<0,001)$, existência de brigas com gritos $(r=-0,542, p<0,001) \mathrm{e}$ a aos problemas emocionais existentes na família $(r=$
$0,532, p<0,001)$. Vale lembrar que esta parte da escala é invertida, assim pode-se apontar que quanto maior a vulnerabilidade social, menor a adaptação, ou seja, maior a incidência de irritação, brigas com gritos e problemas emocionais percebidos pelos entrevistados.

Os escores de autonomia familiar dos IPVS's 1,2 e 3 são significativamente superiores aos dos IPVS's 5 e 6 . O IPVS 4 não possui diferenças significativas com os demais grupos. Os itens da escala mais afetados pelo nível de vulnerabilidade foram em ordem decrescente aqueles relacionados à: privacidade $(r=-0,550, p<0,001)$, autonomia de ser como deseja $(r=-0,521, p<0,001)$ e autonomia para cuidar de si próprio $(r=-0,519, p<$ $0,001)$.

\section{Discussão}

Com os resultados descritivos, observou-se que, de forma geral, os sujeitos seguem o padrão de outras pesquisas apontando a predominância do sexo feminino na população com mais de 60 anos. Em relação à escolaridade, os altos índices de analfabetismo, já esperados segundo dados do IBGE (2007), derivam das desigualdades vividas no cotidiano da sociedade que outrora, estabeleceu papéis ligados ao sexo dentro dos espaços de convivência. Assim, na família, na escola, na igreja e na sociedade, as mulheres eram valorizadas pelo acompanhamento no crescimento dos filhos, dedicação ao lar e ao cônjuge, e os homens, pela formação voltada para o trabalho.

Quanto ao nível sócio-econômico, segundo dados préexistentes da população de São Paulo (ABEP, 2003), foi verificado que a maior parte dos idosos estão nas classes D e E. Neste estudo, a maioria dos idosos (55\%) são da classe $\mathrm{D}$, este dado deve ser apreendido, uma vez que rendas inferiores podem limitar o acesso das pessoas aos cuidados alimentares, sociais e de saúde, comprometendo de forma significativa a qualidade de vida deste grupo.

Em princípio, presumi-se que sujeitos com grau de instrução mais alto dispõem de um espectro mais amplo de recursos para adaptar-se ao meio. Corroborando com Novelli (2006), foi evidenciado que níveis de escolaridade mais elevados levam à adaptação mais satisfatória, afetividade mais consistente e maior autonomia que por sua vez se correlacionam com melhor percepção de qualidade de vida.

A idade mostrou não influenciar negativamente na percepção de qualidade de vida e de suporte familiar. Contudo, a percepção de autonomia relacionou-se fracamente com idades mais elevadas $(r=-0,231, p<0,01)$, ou seja, quanto maior a idade, menor a autonomia e a liberdade do indivíduo dentro da família, principalmente, quando a idade é superior a 76 anos. Isso evidencia a existência de cuidados desnecessários ou providos inadequadamente pelos familiares que cerceiam a qualidade de vida do idoso. 
O fato de morar sozinho não foi um fator determinante de percepções inferiores de suporte familiar. Porém, os idosos que dividiam a residência com pessoas que não eram os filhos (particularmente neste estudo destacamse os netos, genros e noras) tinham escores medianos mais baixos de qualidade de vida. Alinhando-se a este dado, um número maior de filhos ou residentes na mesma casa não elevava os escores medianos de percepção de suporte familiar. Isso evidencia a importância de se equacionar quantidade e qualidade das interações intrafamiliares, além de remeter a questões de subjetividade dos constructos qualidade de vida e suporte familiar.

As relações sociais constituem aspectos fundamentais para o bem-estar dos seres humanos. A família é um sistema complexo composto por vários subsistemas de relações interpessoais, como esposa e marido, pais e filhos, irmãos e irmãs, avós e netos. Cada um destes subsistemas tem regras e limites próprios que regulam a integridade das relações. Os resultados deste estudo corroboram a função de proteção da boa percepção de suporte familiar fomentando a qualidade de vida, e não vinculam o suporte familiar ao número de interações ou de pessoas dividindo o mesmo espaço. Este dado reflete a importância da qualidade e não da quantidade ou configuração dos relacionamentos familiares.

Os escores de qualidade de vida apresentaram-se diretamente proporcionais a renda familiar média, ao nível de instrução e a percepção de suporte familiar (total, afetivo-consistente, de adaptação e autonomia) e, inversamente proporcionais ao fato de se morar com netos, noras e genros. Ainda que este não faça parte dos objetivos da pesquisa, é interessante a observação de que determinados mitos referentes aos arranjos familiares não foram observados neste estudo, e destes destacamos a crença popular que morar com filhas (mulheres) é melhor que morar com filhos (homens).

Os níveis de vulnerabilidade social não são compartimentados e pôde-se verificar, por meio das comparações múltiplas, que para todos os valores medianos de percepção de qualidade de vida e suporte familiar, alguns grupos não se diferiam significativamente entre si. Entretanto, as correlações totais apontam que à medida que o nível de vulnerabilidade social aumenta, diminuem os escores medianos de percepção de suporte familiar e de qualidade de vida.

\section{Conclusões}

A velhice é um momento do ciclo vital no qual a rede social é bastante focada nos familiares e os sujeitos deste estudo apresentavam, em sua maioria, escores elevados de suporte familiar. Isso pode ter ocorrido porque os idosos eram funcionalmente independentes e autônomos e não estavam internados em abrigos ou asilos (situação que pode levar à percepções de abandono ou, ao menos, falta de suporte familiar adequado). Além disso, o viés da desejabilidade social pode ter causado um desvio positivo das avaliações, uma vez que, normas culturais não favorecem críticas sobre familiares com pessoas desconhecidas, neste caso, a pesquisadora. No entanto, este viés estava presente em todos os grupos de vulnerabilidade social, afetando os dados sistematicamente e, portanto, não comprometendo as análises e conclusões comparativas.

Um dos aspectos que comprometem a qualidade de vida do idoso é a dificuldade financeira. Os recursos provenientes da aposentadoria são, às vezes, insuficientes para atender as suas necessidades básicas, obrigando a família a assumir esta responsabilidade. Especialmente neste estudo, grande parte da população de idosos encontra-se entre as classes sociais mais baixas e conseqüentemente em situações de maior vulnerabilidade. A carência financeira limita as possibilidades de escolha por parte dos idosos e dos familiares que, muitas vezes, são forçados a conviverem em um mesmo espaço.

Os laços afetivos, a sensação de pertencimento e a relação de intimidade entre o idoso e a família não se relaciona com a proximidade geográfica entre eles. Coresidir com um idoso pode gerar sobrecarga emocional e situações de estresse se os membros da família não estiverem preparados para a convivência multigeracional. A falta de divisão de trabalho, as dificuldades de adaptação à nova situação, incluindo aspectos culturais intergeracionais e financeiros, a redução de atividades profissionais, sociais e de lazer podem ser condições adversas à qualidade de vida do idoso que é pressionado a fazer concessões. Os contextos familiares de co-residência eram os mais comuns entre os entrevistados, porém não levavam a uma melhor percepção de qualidade de vida e suporte familiar quando comparados aos contextos em que os idosos moravam sozinhos.

Em extremos opostos, existem dois tipos de família, uma distante e outra super-protetora. Na primeira, seus membros estão voltados aos próprios interesses e atividades, contrariamente, na segunda, a proteção assume um caráter extremo, passando a ser asfixiante, minimizando a participação, a independência e a capacidade de resposta. Para que a família seja uma fonte de apoio efetivo para o fomento da qualidade de vida, é preciso que exista um equilíbrio. Os idosos com mais de 76 anos entrevistados apresentaram menor liberdade e autonomia dentro do sistema familiar, este fato evidencia o estereótipo de incapacidade que a sociedade projeta sobre os idosos.

A qualidade de vida dos idosos está potencialmente sob risco não apenas porque existem perdas fisiológicas com o passar do tempo, mas em virtude da maior condição de vulnerabilidade decorrente de baixa escolaridade e condições adversas do meio físico, social ou de questões afetivas. Os vínculos emocionais e as relações familiares não são neutras e este estudo mostra que uma percepção de suporte familiar elevada fomenta a quali- 
dade de vida. Ao planejar serviços públicos e ações de prevenção e controle de agravos ao bem-estar do idoso é necessário contemplar este dado.

Assim, trabalhos que possam identificar quais fatores e que tipos de intervenções favorecem o suporte familiar devem ser alvo de investigações futuras a fim de anunciar o aumento da expectativa de vida não como uma aquisição quantitativa, mas como uma conquista qualitativa em termos de bem-estar, qualidade de vida e satisfação com a família.

\section{Referências}

Associação Brasileira de Empresas de Pesquisa. (2003). Critério de Classificação Econômica Brasil (CCEB). Retrieved November 11, 2008, from http://www.abep.org/codigosguias/ ABEP_CCEB.pdf

Baptista, M. N. (2005). Desenvolvimento do Inventário de Percepção de Suporte Familiar (IPSF): Estudos psicométricos preliminares. PsicoUSF, 10(1), 11-19.

Bernal, I. L. (2003). La familia en la determinación de la salud. Revista Cubana de Salud Pública, 29(1), 48-51.

Busso, G. (2001). La vulnerabilidad social y las políticas sociales a inícios dei sigla XXI: una aproximación a sus potencial idades y limitaciones para los países latinoamericanos. In J. M. P. Cunha, Um sentido para a vulnerabilidade sociodemográfica nas metrópoles paulistas. Retrieved May 04, 2008, from www.nepo.unicamp.br/fotos/vulnerabilidade.pdf

Camarano, A. A., Pasinato, M. T., \& Lemos, V. R. (2007). Cuidados de longa duração para a população idosa: Uma questão de gênero? In A. L. Neri (Ed.), Qualidade de vida na velhice: Enfoque multidisciplinar (pp. 127-149). Campinas, SP: Alínea.

Cattani, R. B., \& Girardon-Perlini, N. M. O. (2004). Cuidar do idoso doente no domicílio na voz de cuidadores familiares. Revista Eletrônica de Enfermagem, 6(2). Retrieved August 14, 2009, from http://www.fen.ufg.br/Revista/revista6_2

Chachamovich, E., Trentini, C. M., \& Fleck, M. P. A. (2007). Qualidade de vida em idosos. In A. L. Neri (Ed.), Qualidade de vida na velhice: Enfoque multidisciplinar (pp. 6181). Campinas, SP: Alínea.

Fleck, M. P. A., Chachamovich, E., \& Trentini, C. M. (2003). Projeto WHOQOL-OLD: Método e resultados de grupos focais no Brasil. Revista de Saúde Pública, 37(6), 793-799.

Fleck, M. P. A., Louzada, S., Xavier, M., Cachamovich, E., Vieira, G, Santos, L., et al. (1999). Aplicação da versão em português do Instrumento de Avaliação de Qualidade de Vida da Organização Mundial da Saúde (WHOQoL-100). Revista de Saúde Pública, 33, 198-205.

Fried, L. P., Tangen, C. M., Walston, J., Newman, A. B., Hirsch, C., Gottdiener, J., et al. (2001). Frailty in older adults: Evidence for a phenotype. Journal of Gerontology, 56(3), 146-156.

Freire, S. A. (2000). Envelhecimento bem-sucedido e bemestar psicológico. In A. L. Neri \& S. A. Freire (Eds.), E por falar em boa velhice (pp. 21-31). Campinas, SP: Papirus.

Fundação SEADE (n.d.) Índice Paulista de Vulnerabilidade Social - IPVS. Espaços e dimensões da pobreza nos municipios do Estado de São Paulo. Retrieved November 12, 2008, from http://www.al.sp.gov.br/web/ipvs/index_ipvs.htm
Gomes, G. V. (1994). Socialização primária: Tarefa familiar? Cadernos de Pesquisa, 91, 54-61.

Inouye, K. (2008). Educação, qualidade de vida e Doença de Alzheimer: Visões de idosos e seus familiares. Dissertação de Mestrado não-publicada, Universidade Federal de São Carlos, SP.

Instituto Brasileiro de Geografia e Estatística. (2007). Contagem da população 2007. Retrieved November 22, 2008, from http://www.ibge.gov.br/home/estatistica/populacao/contagem2007/default.shtm

Karsch, U. M. (2003). Idosos dependentes: Famílias e cuidadores. Cadernos de Saúde Pública, 19(3), 861-866.

Kaztman, R. (2000). Notas sobre la medición de la vulnerabilidad social. 5to. Taller regional. La medición de la pobreza: métodos y aplicaciones. México, DF: Cepal. Retrieved November 12, 2008, from http://www.eclac.cl/ deype/mecovi/docs/TALLER5/24.pdf

Lopes, M. A., \& Bottino, C. M. C. (2002). Prevalência de demência em diversas regiões do mundo. Arquivos de Neuropsiquiatria, 60(1), 61-69.

Martin, A. J., \& Stockler, M. (1998). Quality of life assessment in health care research and practice. Evaluation \& Health Professions, 21, 141-156.

Minayo, M. C. S., Hartz, Z. M. A., \& Buss, P. M. (2000). Qualidade de vida e saúde: Um debate necessário. Ciência \& Saúde Coletiva, 5(1), 7-18.

Neri, A. L. (2005). Redes de suporte social. In A. L. Neri (Ed.), Palavras-chave em gerontologia (pp. 172-175). Campinas, SP: Alínea.

Neri, A. L. (2006). Qualidade de vida no adulto maduro: Interpretações teóricas e evidencias de pesquisa. In A. L. Neri (Ed.), Qualidade de vida na idade madura (pp. 9-56). Campinas, SP: Papirus.

Neri, A. L. (2007). Qualidade de vida na velhice e subjetividade. In A. L. Neri (Ed.), Qualidade de vida na velhice: Enfoque multidisciplinar (pp. 13-59). Campinas, SP: Alínea.

Novelli, M. M. P. C. (2006). Validação da escala de qualidade de vida (QdV-DA) para pacientes com doença de Alzheimer e seus respectivos cuidadores/familiares. Tese de Doutorado não-publicada, Universidade de São Paulo, SP.

Novelli, M. M. P. C., Rovere, H. H., Nitrini, R., \& Caramelli, P. (2005). Cross-cultural adaptation of the quality of life assessment scale on alzheimer disease. Arquivos de NeuroPsiquiatria, 63(2A), 201-206.

Organização Mundial de Saúde. (2005). Envelhecimento ativo: Uma política de saúde (S. Montijo, Trad.). Brasília, DF: Organização Pan-americana da Saúde.

Paschoal, S. M. P., Jacob Filho, W., \& Litvoc, J. (2008). Development of Elderly Quality of Life Index - EqoLI: Item reduction and distribution into dimensions. Clinics, 63(2), 179-188.

Pereira, D. E. C. (2002). Qualidade de vida na terceira idade e sua relação com o trabalho. Dissertação de Mestrado nãopublicada, Universidade Federal de Santa Catarina, Florianópolis, SC.

Ramos, M. P. (2007). Os sintomas depressivos e as relações sociais na terceira idade. Revista do Departamento de Psicologia - UFF, 19(2), 397-410.

Sousa, L., Galante, H., \& Figueiredo, D. (2003). Qualidade de vida e bem-estar dos idosos: Um estudo exploratório na população portuguesa. Revista de Saúde Pública, 37(3), 364-371. 
Souza, C. S. S. C, Mello, I. P., \& Rezende, R. E. S. O. (2008, maio). Avaliação cognitiva, emocional e disponibilidade e adequação de suporte familiar e social de idosos assistidos no ambulatório do Hospital São Julião, Campo Grande, MS. Retrieved November 14, 2008, from http://www.redepsi. com.br/portal/modules/smartsection/item.php?itemid=1144

Teixeira, I. N. D. O. (2006). Definições de fragilidade em idosos: Uma abordagem multiprofissional. Dissertação de Mestrado não-publicada, Universidade Estadual de Campinas, SP.

Williams, L. C. A., \& Aiello, A. L. R. (2004). Empoderamento de famílias. In E. G. Mendes, M. A. Almeida, \& L. C. A. Willians (Eds.), Temas em educação especial: Avanços recentes (pp. 197-202). São Carlos, SP: Editora da Universidade Federal de São Carlos.

World Health Organization Quality of Life Working Group. (1995). The World Health Organization Quality of Life assessment (WHOQOL): Position paper from the World Health Organization. Social Science and Medicine, 10, 14031409. 